\title{
ON THE NUMERICAL SOLUTION OF SINGULAR INTEGRAL EQUATIONS USING SANDIKIDZE'S APPROXIMATON
}

\author{
MOSTEFA NADIR AND D. JEMAL ANTIDZE \\ ${ }^{1}$ Department of Mathematics, University of M'sila, 2800 M'sila, Algeria \\ ${ }^{2}$ InstituteofApplied Mathematics, Tbilisi, Georgia
}

(Rec. 19 March 2004)

\begin{abstract}
The aim of this work is to solve singular integral equations (S.I.E), of Cauchy type on a smooth curve by pieces. This method is based on the approximation of the singular integral of the dominant part [6], where the (S.I.E) is reduced to a linear system of equations and to realize this approach numerically by the means of a program $[3,5]$.
\end{abstract}

\section{INTRODUCTION}

In this work we present a direct method for an approximation solution of a singular integral equation (S.I.E) on a piecewise smooth integration path. Many studies devoted to the numerical procedure are developed for solving (S.I.E) over a finite interval, especially $[-1,1]$. Cauchy type singular integral equations are often encountered in problems of mathematical physics when solving problems in elasticity theory, aerodynamics, electrodynamics and other branches of sciences and technology. Also we note that, the solution of a large class of boundary-value problems in mathematical physics can be reduced to singular integral equations (S.I.E) of the form

$$
f\left(t_{0}\right)=a\left(t_{0}\right) \varphi\left(t_{0}\right)+\frac{b\left(t_{0}\right)}{\pi i} \int_{\Gamma} \frac{\varphi(t)}{t-t_{0}} d t+\frac{1}{\pi i} \int_{\Gamma} k\left(t, t_{0}\right) \varphi(t) d t
$$

where $\Gamma$ is any piecewise smooth path [2], $t_{0}$ and $t$ are points on the curve $\Gamma$, the functions $a(t), b(t)$ and $k\left(t_{0}, t\right)$ are known functions defined on $\Gamma$, satisfying the Hölder condition $H(\alpha)$, $0<\alpha \leq 1$ [2]. Further, anywhere on $\Gamma$ we have $a^{2}(t)-b^{2}(t) \neq 0$.

As it is known, the integral of the dominant part of the above equation (1) exists in the sense of a Cauchy principal value integral for all density $\varphi$ satisfies the Hölder condition $H(\alpha)$ and also exits for all function $\varphi \in L^{2}(\Gamma)$.

The present note is divided into two parts. In the first part, we present a formulation of the quadrature formula for the evaluation of Cauchy type singular integrals proposed by Sanikidze [7], this quadrature formula is based on the classical Lagrange interpolation of the density $\varphi(x)$. In the second part, we present the results of this work concerning the pro- 
gramming and the numerical realization of this approximation; also the estimate of the error of the approximation integral was established. Besides, pointwise convergence of the approximate solutions to an exact solution is obtained [5, 7].

\section{THE QUADRATURE}

A method to proceed is to solve the (S.I.E) by numerical means, like the reduction to a system of linear algebraic equations after the use of an appropriate quadrature rule.

Following [7], we define the approximation $\psi_{\sigma v}(t)$ for the density $\varphi(t)$ by the following expression

$$
\begin{aligned}
& \varphi(t) \cong \psi_{\sigma v}(t)=\varphi\left(t_{0}\right)+\sum_{k=0}^{m-1} l_{\sigma k}(t) \varphi\left(t_{\sigma k}\right) \frac{t-t_{0}}{t_{\sigma k}-t_{0}}+ \\
& +\sum_{k=0}^{m-1} l_{\sigma k}(t) A_{v}\left(t_{0}\right) \frac{t-t_{0}}{t_{0}-t_{\sigma k}}, \quad t \in \tau_{\sigma} \tau_{\sigma+1}, \quad t_{0} \in \tau_{v} \tau_{v+1}
\end{aligned}
$$

where

$$
l_{\sigma, k}(t)=\frac{\omega(t)}{\left(t-t_{\sigma k}\right) \omega^{\prime}\left(t_{\sigma k}\right)}, \quad \omega(t)=\prod_{k=0}^{m-1}\left(t-t_{\sigma k}\right)
$$

and

$$
A_{v}\left(t_{0}\right)=\sum_{k=0}^{m-1} \frac{\omega\left(t_{0}\right)}{\left(t_{0}-t_{v k}\right) \omega^{\prime}\left(t_{v k}\right)} \varphi\left(t_{v k}\right)
$$

Using the classical Lagrange interpolation of the kernel $k\left(t, t_{0}\right)$ and of the density $\varphi(t)$, the regular part of the singular integral equation will be given by

$$
\frac{1}{\pi i} \int_{\Gamma} k\left(t, t_{0}\right) \varphi(t) d t \cong \frac{1}{\pi i} \sum_{\sigma=0}^{n-1} \int_{\tau_{\sigma}} \sum_{\tau_{\sigma+1}}^{m-1} \frac{\omega(t) k\left(t_{\sigma k}, t_{0}\right) \varphi\left(t_{\sigma k}\right)}{\left(t-t_{\sigma k}\right) \omega^{\prime}\left(t_{\sigma k}\right)} d t
$$

The right hand side of (3) can be represented by the expressions of $\alpha_{o k}$,

$$
\alpha_{\sigma k}=\frac{1}{\pi i \omega^{\prime}\left(t_{\sigma k}\right)} \int_{\tau_{\sigma} \tau_{\sigma+1}} \frac{\omega(t)}{t-t_{\sigma k}} d t
$$

and the values of the function product $k\left(t, t_{0}\right) \varphi(t)$ at the points $t_{o k}$ hence, we write

$$
\frac{1}{\pi i} \int_{\Gamma} k\left(t, t_{0}\right) \varphi(t) d t \cong \sum_{\substack{\sigma=0 \\ t_{0} \neq t_{\sigma \hbar}}}^{n-1} \sum_{k=0}^{m-1} \alpha_{\sigma k} k\left(t_{\sigma k}, t_{0}\right) \varphi\left(t_{\sigma k}\right)
$$


Taking into consideration the approximation formulae (2), the singular integral of the dominant part of (S.I.E) (1) takes the form

$$
\begin{gathered}
\frac{1}{\pi i} \int_{\Gamma} \frac{\varphi(t)}{t-t_{0}} d t \cong \frac{1}{\pi i} \int_{\Gamma} \frac{\psi_{\sigma v}\left(\varphi ; t, t_{0}\right)}{t-t_{0}} d t= \\
=\varphi\left(t_{0}\right)+\frac{1}{\pi i} \sum_{\sigma=0}^{n-1}\left(\int_{\tau_{\sigma} \tau_{\sigma+1}} \sum_{k=0}^{m-1} \frac{\omega(t)}{\left(t-t_{\sigma k}\right) \omega^{\prime}\left(t_{\sigma k}\right)} \cdot \frac{A_{v}\left(t_{0}\right)}{t_{0}-t_{\sigma k}} d t-\int_{\tau_{\sigma} \tau_{\sigma+1}} \sum_{k=0}^{m-1} \frac{\omega(t)}{\left(t-t_{\sigma k}\right) \omega^{\prime}\left(t_{\sigma k}\right)} \cdot \frac{\varphi\left(t_{\sigma k}\right)}{t_{0}-t_{\sigma k}} d t\right) .
\end{gathered}
$$

For $\sigma \neq v$, one obtains from the above

$$
\begin{aligned}
& \frac{1}{\pi i} \int_{\Gamma} \frac{\psi_{\sigma v}\left(\varphi ; t, t_{0}\right)}{t-t_{0}} d t=\varphi\left(t_{0}\right)+\sum_{\sigma=0}^{n-1}\left(\sum_{k=0}^{m-1} \frac{\alpha_{\sigma k}}{t_{0}-t_{\sigma k}}\left(A_{v}\left(t_{0}\right)-\varphi\left(t_{\sigma k}\right)\right)=\right. \\
& =\varphi\left(t_{0}\right)+\sum_{\sigma=0}^{n-1}\left(\sum_{k=0}^{m-1} \frac{\alpha_{\sigma k}}{t_{0}-t_{\sigma k}} \times \sum_{\substack{p=0 \\
t_{0} \neq t_{v p}}}^{m-1} \frac{\omega\left(t_{0}\right)}{\left(t_{0}-t_{v p}\right) \omega^{\prime}\left(t_{v p}\right)} \varphi\left(t_{v p}\right)-\varphi\left(t_{\sigma k}\right)\right),
\end{aligned}
$$

and for $\sigma=v$ we can easily see that the expression

$$
\sum_{\sigma=0}^{n-1}\left(\sum_{k=0}^{m-1} \frac{\alpha_{\sigma k}}{t_{0}-t_{\sigma k}}\left(\sum_{p=0}^{m-1} \frac{\omega\left(t_{0}\right)}{\left(t_{0}-t_{v p}\right) \omega^{\prime}\left(t_{v p}\right)} \varphi\left(t_{v p}\right)-\varphi\left(t_{\sigma k}\right)\right)\right)
$$

becomes

$$
\sum_{\sigma=0}^{n-1}\left(\sum_{\substack{k=0 \\ t_{0} \neq t_{\sigma \hbar}}}^{m-1} \frac{\alpha_{\sigma k}}{t_{0}-t_{\sigma k}}\left(\sum_{\substack{p=0 \\ p \neq k, t_{0} \neq t_{\sigma p}, \omega\left(t_{0}\right) \neq 0}}^{m-1} \frac{\omega\left(t_{0}\right)}{\left(t_{0}-t_{\sigma p}\right) \omega^{\prime}\left(t_{\sigma p}\right)} \varphi\left(t_{\sigma p}\right)-\varphi\left(t_{\sigma k}\right)\right)\right)
$$

Finally, for $\sigma, v=0,1, \ldots, n-1$ and $i=0,1, \ldots, m-1$ the expression (5) takes the form

$$
(5)=\sum_{\sigma=0}^{n-1}\left(\sum_{\substack{k=0 \\ i \neq k}}^{m-1} \frac{\alpha_{\sigma k}}{t_{\sigma i}-t_{\sigma k}}\left(\sum_{\substack{p=0 \\ p \neq k, p \neq i}}^{m-1} \frac{\prod_{j=0, j \neq i}^{m-1}\left(t_{\sigma i}-t_{\sigma j}\right)}{\left(t_{\sigma i}-t_{\sigma p}\right) \omega^{\prime}\left(t_{\sigma p}\right)} \varphi\left(t_{\sigma p}\right)-\varphi\left(t_{\sigma k}\right)\right)\right)
$$




\section{THEOREM}

For an arbitrary function $\varphi \in H(\alpha)$ following estimation

$$
\left|\frac{1}{\pi i} \int_{\Gamma} \frac{\varphi(t)}{t-t_{0}} d t-\frac{1}{\pi i} \int_{\Gamma} \frac{\psi_{a v}\left(\varphi ; t, t_{0}\right)}{t-t_{0}} d t\right| \leq \frac{C_{m} \ln n}{n^{\alpha}} \quad(n>1),
$$

is valid, where the constant $C_{m}$ depends only on the curve $\Gamma$ and the Hölder constant of the function $\varphi$.

According to the system of points of subdivisions used in the construction of this approximation $[5,6]$, the singular integral equation (1) can be written as

$$
\begin{aligned}
& f\left(t_{0}\right)=a\left(t_{0}\right) \varphi\left(t_{0}\right)+\frac{b\left(t_{0}\right)}{\pi i} \int_{\Gamma} \frac{\varphi(t)}{t-t_{0}}+\frac{1}{\pi i} \int_{\Gamma} k\left(t, T_{0}\right) \varphi(t) d t \Rightarrow \\
& f\left(t_{0}\right)=\left(a\left(t_{0}\right)+b\left(t_{0}\right)\right) \varphi\left(t_{0}\right)+b\left(t_{0}\right) \sum_{\sigma=0}^{n-1} \sum_{k=0}^{m-1} \frac{\alpha_{\sigma k}}{t_{0}-t_{\sigma k}} \times \\
& \times\left(\sum_{\substack{p=0 \\
t_{0} \neq t_{v, p, \omega\left(t_{0}\right) \neq 0}}}^{\sum^{m-1}} \frac{\omega\left(t_{0}\right)}{\left(t_{0}-t_{v p}\right) \omega^{\prime}\left(t_{v p}\right)} \varphi\left(t_{v p}\right)-\varphi\left(t_{\sigma k}\right)\right)+\sum_{\sigma=0}^{n-1} \sum_{k=0}^{m-1} \alpha_{\sigma k} k\left(t_{\sigma k}, t_{0}\right) \varphi\left(t_{\sigma k}\right)
\end{aligned}
$$

which also, we can write

$$
\begin{aligned}
& g\left(t_{0}\right)=\varphi\left(t_{0}\right)+\frac{1}{a\left(t_{0}\right)+b\left(t_{0}\right)} \sum_{\sigma=0}^{n-1} \sum_{k=0}^{m-1} \frac{b\left(t_{0}\right) \alpha_{\sigma k}}{t_{0}-t_{\sigma k}} \times \\
& \times\left(\sum_{\substack{p=0 \\
t_{0} \neq t_{v, p}, \omega\left(t_{0}\right) \neq 0}}^{m-1} \frac{\omega\left(t_{0}\right)}{\left(t_{0}-t_{v p}\right) \omega^{\prime}\left(t_{v p}\right)} \varphi\left(t_{v p}\right)-\varphi\left(t_{\sigma k}\right)\right)+\sum_{k=0}^{m-1} \alpha_{\sigma k} k\left(t_{\sigma k}, t_{0}\right) \varphi\left(t_{\sigma k}\right)
\end{aligned}
$$

with

$$
g\left(t_{0}\right)=\frac{f\left(t_{0}\right)}{a\left(t_{0}\right)+b\left(t_{0}\right)} .
$$

Replacing the point $t_{0}$ by the system of points $t_{v i}$ of the subdivision between $\tau_{v}$ and $\tau_{v+i}$, we get

$$
\begin{aligned}
& g\left(t_{v i}\right)=\varphi\left(t_{v i}\right)+\frac{1}{a\left(t_{v i}\right)+b\left(t_{v i}\right)} \sum_{\sigma=0}^{n-1} \sum_{k=0}^{m-1} \frac{b\left(t_{v i}\right) \alpha_{\sigma k}}{t_{v i}-t_{\sigma k}} \times \\
& \times\left(\sum_{\substack{p=0 \\
p \neq i, \omega\left(t_{v i}\right) \neq 0}}^{m-1} \frac{\omega\left(t_{v i}\right)}{\left(t_{v i}-t_{v p}\right) \omega^{\prime}\left(t_{v p}\right)} \varphi\left(t_{v p}\right)-\varphi\left(t_{\sigma k}\right)\right)+\sum_{k=0}^{m-1} \alpha_{\sigma k} k\left(t_{\sigma k}, t_{v i}\right) \varphi\left(t_{\sigma k}\right)
\end{aligned}
$$


or, in more detailed form,

$$
\begin{aligned}
& g\left(t_{\sigma i}\right)=\varphi\left(t_{\sigma i}\right)+\frac{1}{a\left(t_{\sigma i}\right)+b\left(t_{\sigma i}\right)} \sum_{\sigma=0}^{n-1}\left(\sum_{\substack{k=0 \\
k \neq i}}^{m-1} \alpha_{\sigma k} k\left(t_{\sigma k}, t_{\sigma i}\right) \varphi\left(t_{\sigma k}\right)+\right. \\
& \left.+\sum_{\substack{k=0 \\
i \neq k}}^{m-1} \frac{b\left(t_{\sigma i}\right) \alpha_{\sigma k}}{t_{\sigma i}-t_{\sigma k}}\left(\sum_{\substack{p=0 \\
p \neq k, p \neq i}}^{m-1} \frac{\prod_{j=0, j \neq i}^{m-1}\left(t_{\sigma i}-t_{\sigma j}\right)}{\left(t_{\sigma i}-t_{\sigma p}\right) \omega^{\prime}\left(t_{\sigma p}\right)} \varphi\left(t_{\sigma p}\right)-\varphi\left(t_{\sigma k}\right)\right)\right)
\end{aligned}
$$

According to this method, we obtain a system of $m \times n$ linear algebraic equations in $m \times n$ unknowns

$$
\begin{aligned}
& g\left(t_{v i}\right)=\varphi\left(t_{v i}\right)+Q(k, m, n, \sigma, v) \varphi\left(t_{\sigma k}\right) \\
& \sigma, v=0,1, \ldots n-1 \text { and } i, k=0,1, \ldots, m-1
\end{aligned}
$$

where

$$
\begin{gathered}
Q(m, n, \sigma, v)=\frac{1}{a\left(t_{v i}\right)+b\left(t_{v i}\right)} \sum_{\sigma=0}^{n-1}\left(\sum _ { \substack { k = 0 \\
t _ { \sigma k } \neq t _ { v i } } } ^ { m - 1 } b ( t _ { v i } ) \left(\sum_{\substack{p=0 \\
\sigma=v}}^{n-1} \sum_{\substack{q=0 \\
t_{p q} \neq t_{v i}}}^{m-1} \frac{\alpha_{p q} l_{\sigma k}\left(t_{v i}\right)}{t_{v i}-t_{p q}}+\right.\right. \\
\left.\left.-\frac{\alpha_{\sigma k}}{t_{v i}-t_{\sigma k}}\right)+\alpha_{\sigma k} k\left(t_{\sigma k}, t_{v i}\right)\right) .
\end{gathered}
$$

\section{NUMERICAL EXPERIMENTS}

In this section we describe some of the numerical experiments performed in solving the singular integral equations (1). In all cases, the curve $\Gamma$ designate the unit circle and we chose the right hand side $f(\mathrm{t})$ in such way that we know the exact solution. This exact solution is used only to show that the numerical solution obtained with our method is correct.

We apply the algorithms described in [5] to solve S.I.E and we present results concerning the accuracy of the calculations; in this numerical experiments it is easily to see that the matrix of the system of algebraic equation given by our approximation is invertible, confirmed in $[5,6]$.

In each table, $\varphi$ represents the exact solution given in the sense of the principal value of Cauchy and $\widetilde{\varphi}$ corresponds to the approximate solution produced by the approximation at points values interpolation [3]. 


\section{Example 1}

We start with the easiest type, without the regular part

$$
t_{0}\left(t_{0}+2\right) \varphi\left(t_{0}\right)+\frac{\left(t_{0}+2\right)\left(t_{0}-6\right)}{\pi i} \int_{\Gamma} \frac{\varphi(t)}{\left(t-t_{0}\right)} d t=-\frac{6}{t_{0}}
$$

where the function $f\left(t_{0}\right)$ is chosen so that the solution $\varphi(t)$ is given by

$$
\varphi(t)=\frac{-2 t^{2}+8 t+12}{4 t\left(t^{2}-t-6\right)}
$$

\begin{tabular}{cccc}
\hline$N$ and $M$ & $\|\varphi-\tilde{\varphi}\|_{1}$ & $\|\varphi-\widetilde{\varphi}\|_{2}$ & $\|\varphi-\tilde{\varphi}\|_{\infty}$ \\
\hline 10 and 4 & $1.6154085 \mathrm{E}-03$ & $7.0876902 \mathrm{E}-04$ & $4.3220818 \mathrm{E}-04$ \\
10 and 3 & $8.2964720 \mathrm{E}-03$ & $3.5810359 \mathrm{E}-03$ & $2.0005703 \mathrm{E}-03$ \\
\hline$N$ and $M$ & $\|\varphi-\widetilde{\varphi}\|_{1}$ & $\|\varphi-\widetilde{\varphi}\|_{2}$ & $\|\varphi-\widetilde{\varphi}\|_{\infty}$ \\
\hline 9 and 4 & $2.6929409 \mathrm{E}-03$ & $1.1711853 \mathrm{E}-03$ & $7.0869923 \mathrm{E}-04$ \\
9 and 3 & $1.2383505 \mathrm{E}-02$ & $5.3870520 \mathrm{E}-03$ & $3.0545730 \mathrm{E}-03$ \\
\hline
\end{tabular}

Example 2

Consider the singular integral equation

$$
-t_{0}\left(t_{0}-2\right) \varphi\left(t_{0}\right)-\frac{t_{0}\left(t_{0}+5\right)}{t-t_{0}} \int_{\Gamma} \frac{\varphi(t)}{t-t_{0}} d t+\frac{1}{\pi i} \int_{\Gamma} \frac{t_{0}(t+2)}{t} \varphi(t) d t=\frac{t_{0}}{t_{0}+2}
$$

where the function $f\left(t_{0}\right)$ is chosen so that the solution $\varphi(\mathrm{t})$ is given by

$$
\varphi(t)=\frac{1}{t+2}
$$

\begin{tabular}{cccc}
\hline$N$ and $M$ & $\|\varphi-\tilde{\varphi}\|_{1}$ & $\|\varphi-\tilde{\varphi}\|_{2}$ & $\|\varphi-\tilde{\varphi}\|_{\infty}$ \\
\hline 10 and 4 & $3.2980263 \mathrm{E}-02$ & $1.2481871 \mathrm{E}-02$ & $5.8662486 \mathrm{E}-03$ \\
10 and 3 & $3.6064602 \mathrm{E}-02$ & $1.5459331 \mathrm{E}-02$ & $8.1108334 \mathrm{E}-03$ \\
\hline$N$ and $M$ & $\|\varphi-\widetilde{\varphi}\|_{1}$ & $\|\varphi-\widetilde{\varphi}\|_{2}$ & $\|\varphi-\widetilde{\varphi}\|_{\infty}$ \\
\hline 9 and 4 & $4.2431947 \mathrm{E}-02$ & $1.5598458 \mathrm{E}-02$ & $6.9491039 \mathrm{E}-03$ \\
9 and 3 & $4.2586692 \mathrm{E}-02$ & $1.7830163 \mathrm{E}-02$ & $9.0550194 \mathrm{E}-03$ \\
\hline
\end{tabular}




\section{CONCLUSIONS}

We have considered a numerical solution of singular integral equations and have presented an efficient scheme to compute these singular integrals. The essential idea is to find a combination of functions which approximate the function density and to use it to remove integrable singularities. The regular part, being the remaining integrand, is well behaved and poses no serious numerical problem. Typical examples taken from the literature, with known closed form solutions, were used to illustrate the stability and convergence of the approach. The stability of the numerical solution was verified by comparing the analytical and numerical solutions; they show a good agreement.

\section{Reference}

[1] D. J. Antidze, On the approximate solution of singular integral equations, Seminar of Institute of Applied Mathematics, 1975, Tbilissi.

[2] N. I. Muskhelishvili, Singular integral equations, "Nauka" Moscow, 1968, English transl, of 1sted Noordhoff, 1953; reprint, 1972.

[3] M. Nadir, Problemes aux limites qui se reduisent aux equations integrales de Fredholm, Seminaire de l'Institut de Mathematiques et Informatique, 1985, Annaba.

[4] M. Nadir, Operateurs integraux et bases d'ondelettes, Far East J. Sci. 6(6) 977-995 (1998).

[5] M. Nadir, On the approximation of theHilbert transform, Far East J. App. Math. 1, 71-786 (2003).

[6] J. Sanikidze, On approximate calculation of singular line integral, Seminar of Institute of Applied Mathematics, 1970, Tbilissi

[7] J. Sanikidze, Approximate solution of singular integral equations in the case of closed contours of integration, Seminar of Institute of Applied Mathematics, 1971, Tbilissi. 